\title{
Échographie et prise en charge de l'arrêt cardiaque
}

\section{Ultrasound and management of cardiac arrest}

\author{
S. Pommet $\cdot$ P.-G. Claret $\cdot$ J.-E. de La Coussaye $\cdot$ X. Bobbia \\ Reçu le 9 janvier 2018; accepté le 14 mars 2018 \\ (C) SFMU et Lavoisier SAS 2018
}

Résumé L'arrêt cardiorespiratoire (ACR) est une situation fréquente pour les structures d'urgences et de réanimation, et l'utilisation de l'échographie y est recommandée par les sociétés savantes. Elle doit impérativement se faire sans augmentation des temps d'interruption du massage cardiaque externe. Après formation, elle permet de détecter rapidement et facilement la présence d'une contractilité myocardique qui est un élément pronostique important pouvant aider dans la décision d'arrêter la RCP. Le recours à des protocoles bien établis avec des équipes entraînées permet d'éviter une interruption prolongée de la RCP. Cependant, aucune étude humaine de grande ampleur n'a permis de décrire de manière fiable pendant l'ACR la sémiologie échographique des causes curables que constituent l'hypovolémie, l'embolie pulmonaire, la tamponnade et le pneumothorax compressif.

Mots clés Asystolie $\cdot$ Arrêt cardiaque $\cdot$ Échographie

\begin{abstract}
Cardiorespiratory arrest (CA) is a common situation for emergency and resuscitation structures and the use of ultrasound is recommended. It must be done without increasing the external cardiac massage interruption times. After training, it allows to detect quickly and easily the presence of myocardial contractility, which is an important prognostic element that can help in the decision to stop cardiopulmonary resuscitation (CPR). The use of well-established protocols with trained teams avoids a prolonged interruption of CPR. However, no large-scale human studies have been able to reliably describe the echographic semiology of the
\end{abstract}

S. Pommet $(\triangle) \cdot$ P.-G. Claret $\cdot$ J.-E. de La Coussaye $\cdot$ X. Bobbia Pôle anesthésie-réanimation-douleur-urgences, CHU de Nîmes, place du Professeur-Robert-Debré, F-30029 Nîmes cedex 09, France

e-mail : stephane.pommet@chu-nimes.fr

J.-E. de La Coussaye

Université Montpellier-Nîmes,

2, rue École-de-Médecine,

F-34060 Montpellier, France curable causes of hypovolemia, pulmonary embolism, tamponade, and compressed pneumothorax during CA.

Keywords Asystole $\cdot$ Cardiac arrest $\cdot$ Echocardiography

\section{Introduction}

L'arrêt cardiorespiratoire (ACR) est une situation fréquente pour les structures d'urgences et de réanimation. En 2016, 13952 ACR préhospitaliers ont été recensés en France. La reprise d'une activité cardiaque spontanée (RACS) était observée dans $19 \%$ des cas, et seulement $15 \%$ ont été admis à l'hôpital avec une activité cardiaque spontanée [1]. Aux États-Unis, la survie globale est de $8 \%$ et s'élève à $28 \%$ lorsqu'un rythme choquable est présent [2]. Dans les ACR intrahospitaliers, les rythmes choquables sont rencontrés dans $32 \%$ des cas avec une survie de $28 \%$. Ce pourcentage de survie s'élève à $50 \%$ lorsqu'un rythme choquable est présent à la prise en charge [3]. Différents scores pronostiques de l'ACR sont proposés dans la littérature : l'OHCA score, le CAHP score et, plus récemment, le TTM Risk Score [4-6]. L'absence de rythme choquable, les durées de no-flow et low-flow sont des variables communes aux trois. Cependant, aucun de ces scores n'intègre l'échographie cardiaque.

\section{Recommandations concernant les ACR et l'échographie}

En 2014, les recommandations américaines sur l'utilisation de l'échographie cardiaque suggéraient dans le cas particulier de l'ACR [7] :

- d'avoir recours à des protocoles de prise en charge spécifiques dans le cas de rythme non choquable (grade IB) ;

- que la voie sous-xiphoïdienne était la coupe de prédilection (grade IB) ; 
- que l'échographie cardiaque permettait de rechercher une contractilité myocardique (grade IA);

- que l'échographie cardiaque était plus sûre que l'électrocardiogramme pour déterminer la présence d'une activité cardiaque (grade IA) ;

- que l'échographie cardiaque pouvait changer la prise en charge de l'ACR (grade IA) ;

- que l'échographie cardiaque avait un intérêt pronostique (grade IB) ;

- que l'échographie cardiaque était plus précise que l'examen clinique pour déterminer le mécanisme de l'ACR (grade IB) et la fonction cardiaque (grade IA) ;

- que l'échographie cardiaque n'améliorait pas le devenir du patient.

En 2015, les recommandations européennes sur la prise en charge de l'ACR n'évoquaient que brièvement la place de l'échographie [8]. Il était supposé que la mise en place de protocoles spécifiques d'échographie pendant la réanimation cardiopulmonaire (RCP) pouvait permettre d'identifier des causes réversibles telles que l'embolie pulmonaire (EP), la tamponnade, l'hypovolémie et le pneumothorax. Ainsi, pour minimiser les interruptions de la RCP, les auteurs recommandaient la réalisation d'une coupe sous-xiphoïdienne par des praticiens entraînés, avec acquisition des images pendant les dix secondes d'analyse du rythme du patient. Il était précisé que l'absence de mouvement cardiaque était un facteur indépendant de mauvais pronostic sans précision sur la sensibilité et la spécificité.

\section{Principaux protocoles d'échographie lors des ACR}

\section{Protocole FEER (Focused Echocardiographic Evaluation in Resuscitation) [9]}

En 2007, Breitkreutz et al. proposaient un algorithme incluant l'échocardiographie dans l'ACR avec le protocole FEER. L'échographie cardiaque était réalisée en cas de rythme non choquable après cinq cycles de RCP (Fig. 1). Le but de l'échocardiographie était de dépister la présence ou l'absence de mouvement cardiaque, de rechercher une hypo- ou une hypercontractilité, une dilatation du ventricule droit ou un épanchement péricardique. Ce protocole fut rebaptisé FEEL (Focused Echocardiographic Evaluation in Life support) en 2010, mais en gardant le même principe [10].

\section{Protocole CAUSE (Cardiac Arrest Ultrasound Exam) [11]}

En 2008, Hernandez et al. proposaient un algorithme intégrant l'échographie cardiaque et pulmonaire et préconisaient une coupe quatre cavités, dans un premier temps en souscostal et si besoin en apical. Les modalités opérationnelles n'étaient pas définies (Fig. 2).

\section{Protocole PEA (Pleuro- \\ pulmonary-Epigastric-Abdominal) $[12,13]$}

En 2010, Testa et al. proposaient d'étendre l'échographie cardiaque et pulmonaire à l'évaluation de la veine cave inférieure, à la recherche d'épanchement abdominal, d'anévrisme aortique ou de thrombose veineuse profonde. La séquence d'acquisition des coupes était laissée à l'appréciation du médecin, même s'il était recommandé de commencer par l'échographie cardiaque.

\section{Protocole SHoC (Sonography in Hypotension and Cardiac Arrest) [14]}

Récemment, l'International Federation for Emergency Medicine a présenté un algorithme suivant la règle des « $4 \mathrm{~F}$ » (fluid-form-function-filling) et s'inspirant des protocoles présentés ci-dessus. L'analyse échographique est stratifiée en trois étapes d'analyse (Fig. 3) : analyse de base pour tous les patients en ACR ; analyse supplémentaire si le temps le permet et si nécessité d'informations complémentaires ; analyse additionnelle guidée par la clinique et les circonstances. Le protocole inclut une check-list opérationnelle à respecter :

- l'équipe doit être formée ;

- l'appareil d'échographie doit être préparé avant la prise en charge ;

- les images sont enregistrées pendant les dix secondes durant l'analyse du rythme ;

- les images enregistrées sont analysées pendant la RCP ;

- prise de décision en fonction de l'analyse échographique ;

- analyses complémentaires si besoin ;

- prise en charge post-RACS.

\section{Intérêt clinique et pratique de l'échographie lors des ACR}

\section{Rechercher une contractilité myocardique}

En 2001, Blaivas et al. étaient les premiers à montrer que l'absence de contractilité à l'échographie était un marqueur prédictif de mortalité avec une valeur prédictive positive de $100 \%$, une valeur prédictive négative de $58 \%$ et un rapport de vraisemblance négatif à 0,17 [15]. Ces données obtenues en intrahospitalier se confirment en préhospitalier bien que la valeur prédictive positive soit plus faible (97\%) [16]. Plus récemment, une méta-analyse incluant 1695 patients a 


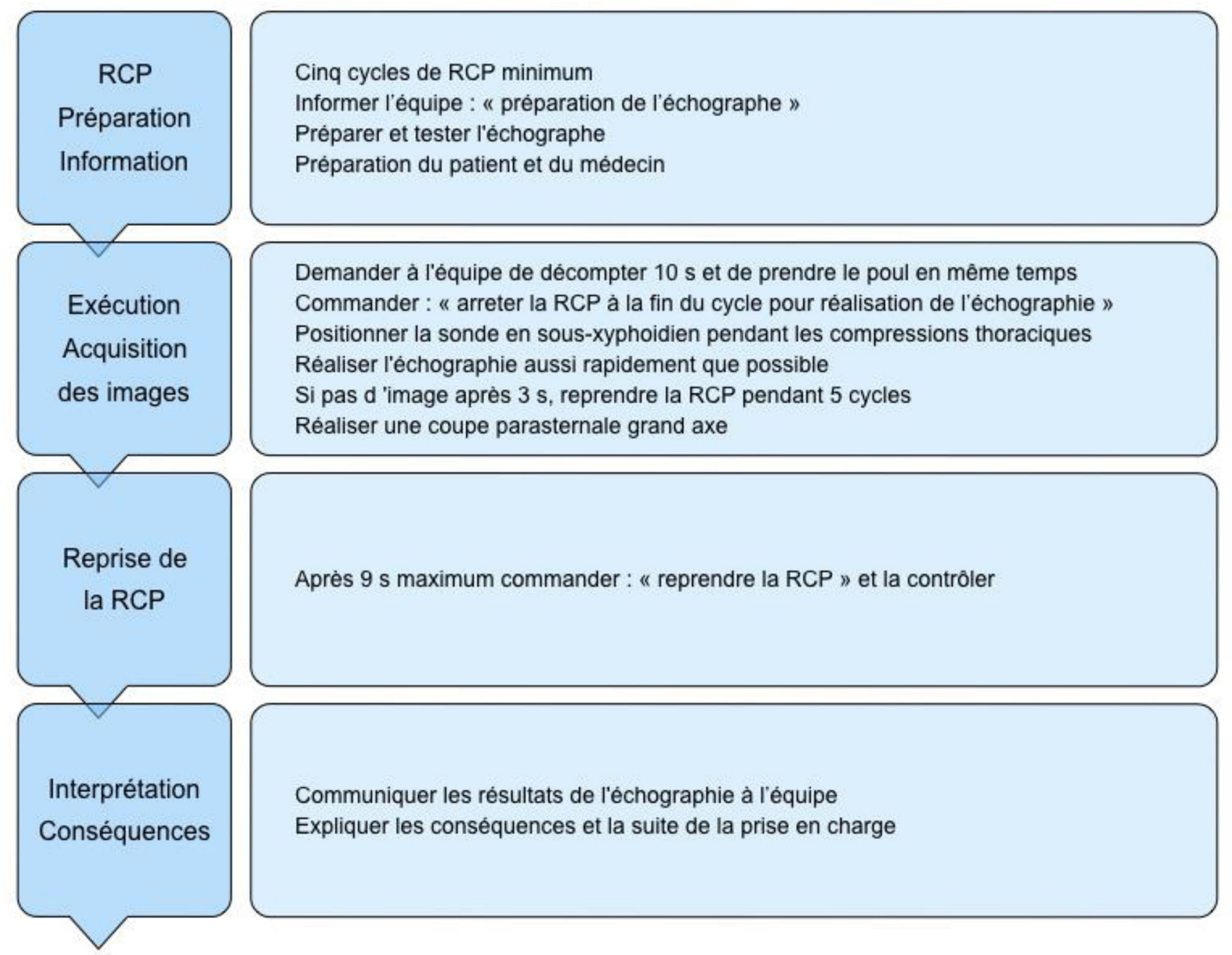

Fig. 1 Protocole FEER (Focused Echocardiographic Evaluation in Resuscitation). RCP : réanimation cardiopulmonaire

objectivé que la mise en évidence d'une contractilité myocardiaque à l'échographie était prédictive d'une RACS avec une sensibilité de $95 \%$, une spécificité de $80 \%$, un rapport de vraisemblance positif de 4,8 et un rapport de vraisemblance négatif de 0,06 [17]. Ces résultats sont superposables à la méta-analyse de 2012 réalisée par Blyth et al. [18]. Dans une étude prospective préhospitalière de 2010, Breitkrutz et al. montraient que $75 \%$ des patients présentent une activité électrique sans pouls, et $35 \%$ des patients en asystolie présentent des mouvements cardiaques à l'échographie [10]. Les auteurs montrent également que la présence de mouvements cardiaques est associée à un meilleur pronostic avec une survie à l'admission de 73 vs $13 \%$.

\section{Rechercher une cause réversible}

Le diagnostic étiologique précoce de l'ACR améliore de $19 \%$ la survie à 30 jours, surtout si la cause est extracardiaque [19].
Il est donc important de pouvoir identifier précocement la cause de l'ACR afin d'adapter la prise en charge et de traiter les causes réversibles définies à la règle des « $4 \mathrm{H}$ » (pour hypoxie, hypovolémie, hypo-/hyperkaliémie et hypothermie) et « $4 \mathrm{~T}$ » (pour tamponnade, thrombus coronaire ou pulmonaire, toxique et tension pour les pneumothorax) [13]. Cependant, Milne et al. ont montré récemment qu'au cours de l'ACR les seules anomalies échographiques rencontrées étaient des troubles de la contractilité du ventricule gauche (45\%) ou une dysfonction valvulaire (39\%) [20]. À ce jour, il n'existe pas d'étude in vivo sur la fiabilité diagnostique de l'échographie cardiaque pendant l'ACR.

\section{Évaluer les cavités droites}

L'ACR post-EP survient lorsqu'il y a obstruction de plus des deux tiers du lit vasculaire pulmonaire [21]. Elle est responsable de $4,8 \%$ des ACR [22]. La dilatation des cavités 


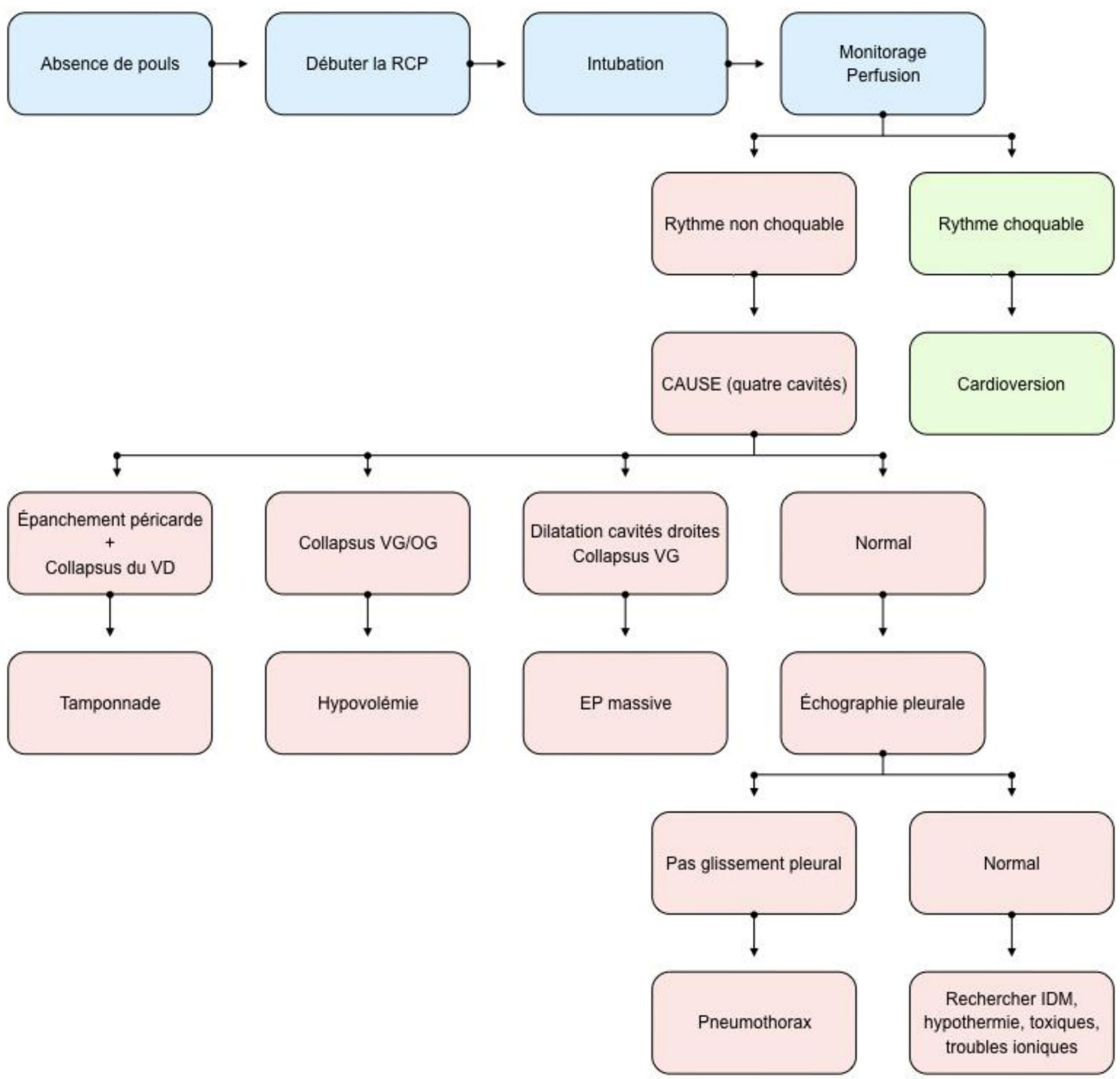

Fig. 2 Protocole CAUSE (Cardiac Arrest Ultrasound Exam). VD : ventricule droit ; VG ventricule gauche ; OG : oreillette gauche ; EP : embolie pulmonaire ; IDM : infarctus du myocarde

cardiaques droites avec une hyperkinésie du ventricule gauche et la présence d'un septum paradoxal (signe du D) sont des éléments évocateurs d'EP chez les patients instables et posent l'indication de fibrinolyse si le patient n'est pas transportable à la tomodensitométrie [23]. Si ces signes sont souvent présents et facilement reconnaissables dans la phase précédant l'ACR, une dilatation des cavités droites survient précocement après. Chez le porc, le ventricule droit se dilate dès la première minute après l'apparition d'une fibrillation ventriculaire, alors que le ventricule gauche se dilate plus tardivement $[24,25]$. Cette dilatation persiste pendant la réanimation, y compris en cas d'ACR hypoxique et d'EP, même si elle semble plus marquée dans ce dernier cas [26]. Ainsi, dans le cas de l'ACR avec absence de mouvement cardiaque, la dilatation seule des cavités droites n'est pas toujours synonyme d'EP, sauf en cas de mise en évidence d'un thrombus ou d'une prise en charge très précoce (ACR intrahospitalier ou devant une équipe médicale) $[27,28]$. Par opposition, l'absence de signe de cour droit permettrait d'exclure l'EP comme diagnostic étiologique [29]. 


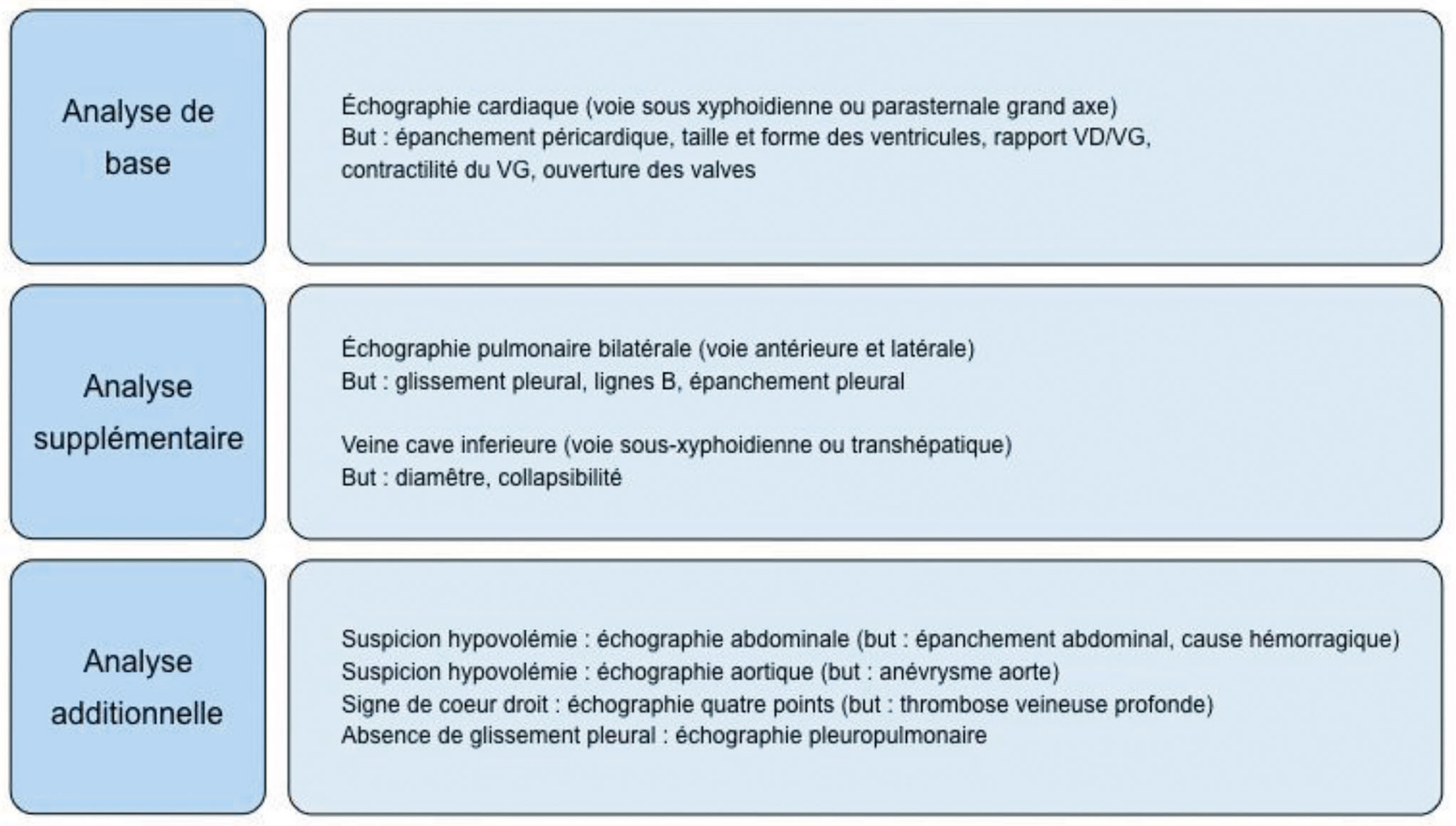

Fig. 3 Protocole SHoC (Sonography in Hypotension and Cardiac Arrest)

\section{Rechercher une hypovolémie}

En l'absence d'activité mécanique cardiaque, chez le porc, une dilatation des cavités droites est observée dans les ACR secondaires à une hypovolémie, à une hyperkaliémie ou à une arythmie primitive [30]. Cependant, en présence d'activité mécanique, de nombreux auteurs suggèrent qu'un ventricule gauche hyperkinétique, collabé, associé à une veine cave inférieure collabée doit conduire à un remplissage agressif et à rechercher une cause d'hypovolémie [21,31,32].

\section{Rechercher une tamponnade}

L'échographie est l'examen de référence dans le diagnostic de tamponnade. Elle recherche un épanchement péricardique, une " danse du cœur » (swinging heart), une compression des cavités droites, une dilatation de la veine cave inférieure, une variabilité respiratoire des flux transvalvulaires et de la taille des cavités cardiaques [33]. Dans l'épanchement péricardique aigu, des volumes modérés d'épanchements pourront entraîner un retentissement hémodynamique [34]. Les signes de compression des cavités droites et la contextualisation clinique seront alors primordiaux pour établir un diagnostic étiologique.

Enfin, dans l'ACR traumatique, l'échographie cardiaque diminue la durée de prise en charge préhospitalière, diminue le nombre de thoracotomies non indiquées, le nombre de transfusions, ainsi que le coût de prise en charge des ACR traumatiques qui décèdent [35].

\section{Rechercher un pneumothorax}

L'échographie pleurale permet d'exclure un pneumothorax dans $100 \%$ des cas si le glissement pleural est présent [36]. Par contre, l'absence de glissement pleural n'est pas synonyme de pneumothorax, surtout chez le patient critique, puisque la spécificité chute de $91 \%$ dans la population générale à $78 \%$ chez le patient réanimatoire et même à $60 \%$ en cas de syndrome de détresse respiratoire aiguë (SDRA). En cas de défaillance respiratoire aiguë, la valeur prédictive positive du glissement pleural n'est que de $27 \%$ [37]. En cas d'ACR, d'intubation sélective ou œsophagienne, de SDRA, d'atélectasie, de fibrose sévère, de paralysie phrénique ou de mauvais réglage de l'appareil, le glissement pleural peut être absent et peut conduire à un mauvais diagnostic. Le bon sens clinique reste donc primordial. Il est évident que l'échographie pleurale devra être réalisée après intubation (la ventilation au masque ne permettant pas d'obtenir des volumes suffisants pour bien visualiser le glissement pleural).

\section{Faisabilité de l'échographie}

Aux États-Unis, des secouristes (paramedics) ayant validé une formation de trois heures en échographie transthoracique 
sont capables d'acquérir des coupes satisfaisantes dans $89 \%$ des cas et de différencier la présence ou non d'une activité cardiaque dans $100 \%$ des cas [38]. La mise en place de formation courte permet de modifier rapidement et durablement les pratiques. Preston et al. rapportent une augmentation de 4 à $20 \%$ (et même $31 \%$, six mois après la fin de l'étude) d'utilisation de l'échographie dans l'ACR après une formation d'un jour [39]. La réalisation d'analyse échocardiographique plus complète pose le problème de l'expérience du praticien puisqu'elle constitue le seul critère indépendant d'interprétabilité des images en préhospitalier [40,41]. Dans l'étude préhospitalière de Breitkrutz et al., réalisée par des équipes expérimentées et formées au protocole FEER, seulement $50 \%$ des images enregistrées étaient de bonne qualité, alors qu'une modification thérapeutique était observée dans $89 \%$ des cas [10].

\section{Contraintes de l'utilisation de l'échographie dans l'ACR}

Malgré les nombreux protocoles décrits pour permettre d'intégrer le geste échographique dans la prise en charge standardisée des ACR, deux études récentes montrent les contraintes de cette technique $[42,43]$. Ces études observationnelles montrent que l'utilisation de l'échographie durant l'ACR augmente les temps d'interruptions de massage cardiaque externe (MCE). Si aucun effet n'est montré sur la proportion de RACS ou de survie, ces études, de très faible effectif, ont le mérite de mettre en avant le risque lié à l'utilisation de la technique. Aucune précision sur l'utilisation de protocoles standardisés et la formation des professionnels n'est apportée. Montrer que des soignants dont on ne connaît pas le niveau de formation peuvent faire mal n'est pas suffisant pour remettre en question l'intérêt de la technique, mais permet de rappeler l'enjeu de son développement qui est de ne pas augmenter les temps d'interruption du MCE grâce à des équipes formées.

\section{Conclusion}

Dans l'ACR, l'utilisation de l'échographie est recommandée par les sociétés savantes. Elle doit impérativement se faire sans augmentation des temps d'interruption du MCE. Après formation, elle permet de détecter rapidement et facilement la présence d'une contractilité myocardique qui est un élément pronostique important pouvant aider dans la décision d'arrêter la RCP. Le recours à des protocoles bien établis avec des équipes entrainées permet d'éviter une interruption prolongée de la RCP. L'intérêt de l'échographie dans le diagnostic étiologique de l'ACR nécessite probablement de meilleures compétences échographiques en intégrant l'échographie cardiaque, pleuropulmonaire et abdominale de manière séquentielle comme le propose le protocole SHoC. Aucune étude humaine de grande ampleur n'a permis de décrire de manière fiable, pendant l'ACR, la sémiologie échographique des causes curables que constituent l'hypovolémie, l'EP, la tamponnade et le pneumothorax compressif. Des études prospectives sont donc nécessaires afin de déterminer si les anomalies échographiques constatées chez les patients choqués sont reproductibles dans l'ACR et si l'emploi de l'échographie améliore la morbimortalité.

Liens d'intérêts : les auteurs déclarents ne pas avoir de liens d'intérêts.

\section{Références}

1. RéAC (2018) Registre électronique des arrêts cardiaques. http:// registreac.org/ (Dernier accès le 9 janvier 2018)

2. Chan PS, McNally B, Tang F, Kellermann A (2014) Recent trends in survival from out-of-hospital cardiac arrest in the United States. Circulation 130:1876-82

3. Al-Dury N, Rawshani A, Israelsson J, et al (2017) Characteristics and outcome among 14,933 adult cases of in-hospital cardiac arrest: a nationwide study with the emphasis on gender and age. Am J Emerg Med 35:1839-44

4. Adrie C, Cariou A, Mourvillier B, et al (2006) Predicting survival with good neurological recovery at hospital admission after successful resuscitation of out-of-hospital cardiac arrest: the OHCA score. Eur Heart J 27:2840-5

5. Martinell L, Nielsen N, Herlitz J, et al (2017) Early predictors of poor outcome after out-of-hospital cardiac arrest. Crit Care 13:96

6. Maupain C, Bougouin W, Lamhaut L, et al (2016) The CAHP (Cardiac Arrest Hospital Prognosis) score: a tool for risk stratification after out-of-hospital cardiac arrest. Eur Heart J 7:3222-8

7. Via G, Hussain A, Wells M, et al (2014) International evidencebased recommendations for focused cardiac ultrasound. J Am Soc Echocardiogr 27:683.e1-683.e33

8. Soar J, Nolan JP, Böttiger BW, et al (2015) European Resuscitation Council Guidelines for resuscitation 2015: Section 3. Adult advanced life support. Resuscitation 95:100-47

9. Breitkreutz R, Walcher F, Seeger FH (2007) Focused echocardiographic evaluation in resuscitation management: concept of an advanced life support-conformed algorithm. Crit Care Med 35: S150-S161

10. Breitkreutz R, Price S, Steiger HV, et al (2010) Focused echocardiographic evaluation in life support and peri-resuscitation of emergency patients: a prospective trial. Resuscitation 81:1527-33

11. Hernandez C, Shuler K, Hannan H, et al (2008) CAUSE: Cardiac arrest ultra-sound exam - a better approach to managing patients in primary non-arrhythmogenic cardiac arrest. Resuscitation 76:198-206

12. Testa A (2008) Manuale di ecografia clinica in urgenza. Verduci Ed., Rome

13. Testa A, Cibinel GA, Portale G, et al (2010) The proposal of an integrated ultrasonographic approach into the ALS algorithm for cardiac arrest: the PEA protocol. Eur Rev Med Pharmacol Sci 14:77-88

14. Atkinson P, Bowra J, Milne J, et al (2017) International Federation for Emergency Medicine Consensus Statement: sonography in hypotension and cardiac arrest (SHoC): an international consensus on the use of point of care ultrasound for undifferentiated hypotension and during cardiac arrest. CJEM 19:459-70 
15. Blaivas M, Fox JC (2001) Outcome in cardiac arrest patients found to have cardiac standstill on the bedside emergency department echocardiogram. Acad Emerg Med 8:616-21

16. Aichinger G, Zechner PM, Prause G, et al (2012) Cardiac movement identified on prehospital echocardiography predicts outcome in cardiac arrest patients. Prehosp Emerg Care 16:251-5

17. Tsou PY, Kurbedin J, Chen YS, et al (2017) Accuracy of pointof-care focused echocardiography in predicting outcome of resuscitation in cardiac arrest patients: a systematic review and metaanalysis. Resuscitation 114:92-9

18. Blyth L, Atkinson P, Gadd K, Lang E (2012) Bedside focused echocardiography as predictor of survival in cardiac arrest patients: a systematic review. Acad Emerg Med 19:1119-26

19. Bergum D, Haugen BO, Nordseth T, et al (2015) Recognizing the causes of in-hospital cardiac arrest--A survival benefit. Resuscitation 97:91-6

20. Milne J, Atkinson P, Lewis D, et al (2016) Rates of abnormal findings in undifferentiated hypotension and during cardiac arrest as a basis for consensus on a hierarchical point of care ultrasound protocol. Cureus 8:e564

21. Price S, Uddin S, Quinn T (2010) Echocardiography in cardiac arrest. Curr Opin Crit Care 16:211-5

22. Kürkciyan I, Meron G, Sterz F, et al (2000) Pulmonary embolism as a cause of cardiac arrest: presentation and outcome. Arch Intern Med 160:1529-35

23. Kucher N, Luder CM, Dörnhöfer T, et al (2003) Novel management strategy for patients with suspected pulmonary embolism. Eur Heart J 24:366-76

24. Berg RA, Sorrell VL, Kern KB, et al (2005) Magnetic resonance imaging during untreated ventricular fibrillation reveals prompt right ventricular overdistention without left ventricular volume loss. Circulation 111:1136-40

25. Steen S, Liao Q, Pierre L, et al (2003) The critical importance of minimal delay between chest compressions and subsequent defibrillation: a haemodynamic explanation. Resuscitation 58:249-58

26. Aagaard R, Caap P, Hansson NC, et al (2017) Detection of pulmonary embolism during cardiac arrest-ultrasonographic findings should be interpreted with caution. Crit Care Med 45:e695-e702

27. MacCarthy P, Worrall A, McCarthy G, Davies J (2002) The use of transthoracic echocardiography to guide thrombolytic therapy during cardiac arrest due to massive pulmonary embolism. Emerg Med J 19:178-9

28. Chapoutot L, Nazeyrollas P, Metz D, et al (1996) Floating right heart thrombi and pulmonary embolism: diagnosis, outcome and therapeutic management. Cardiology 87:169-74

29. Torbicki A, Perrier A, Konstantinides S, et al (2008) Guidelines on the diagnosis and management of acute pulmonary embolism: The
Task Force for the Diagnosis and Management of Acute Pulmonary Embolism of the European Society of Cardiology (ESC). Eur Heart J 29:2276-315

30. Aagaard R, Granfeldt A, Bøtker MT, et al (2017) The right ventricle is dilated during resuscitation from cardiac arrest caused by hypovolemia: a porcine ultrasound study. Crit Care Med 45: e963-e970

31. Zafiropoulos A, Asrress K, Redwood S, et al (2014) Critical care echo rounds: echo in cardiac arrest. Echo Res Pract 1:D15-D21

32. Hendrickson RG, Dean AJ, Costantino TG (2001) A novel use of ultrasound in pulseless electrical activity: the diagnosis of an acute abdominal aortic aneurysm rupture. J Emerg Med 21:141-44

33. Tayal VS, Kline JA (2003) Emergency echocardiography to detect pericardial effusion in patients in PEA and near-PEA states. Resuscitation 59:315-18

34. Spodick DH (2003) Acute cardiac tamponade. N Engl J Med 349:684-90

35. Ferrada P, Wolfe L, Anand RJ, et al (2014) Use of limited transthoracic echocardiography in patients with traumatic cardiac arrest decreases the rate of nontherapeutic thoracotomy and hospital costs. J Ultrasound Med 33:1829-32

36. Lichtenstein D (1997) Pulmonary echography: a method of the future in emergency medicine and resuscitation. Rev Pneumol Clin 53:63-8

37. Lichtenstein D (2008) Échographie pulmonaire en réanimation et aux urgences. Réanimation 17:722-30

38. Rooney KP (2016) Pre-hospital assessment with ultrasound in emergencies: implementation in the field. World J Emerg Med 7:117

39. Preston HM, Collins AST, Reed MJ, Connolly J (2015) A description of echocardiography in life support use during cardiac arrest in an Emergency Department before and after a training programme. Eur J Emerg Med 22:426-29

40. Bobbia X, Pradeilles C, Claret PG, et al (2015) Does physician experience influence the interpretability of focused echocardiography images performed by a pocket device? Scand J Trauma Resusc Emerg Med 23:52

41. Niendorff DF, Rassias AJ, Palac R, et al (2005) Rapid cardiac ultrasound of inpatients suffering PEA arrest performed by nonexpert sonographers. Resuscitation 67:81-7

42. Clattenburg EJ, Wroe P, Brown S, et al (2017) Point-of-care ultrasound use in patients with cardiac arrest is associated prolonged cardiopulmonary resuscitation pauses: a prospective cohort study. Resuscitation 122:65-8

43. Huis In't Veld MA, Allison MG, Bostick DS, et al (2017) Ultrasound use during cardiopulmonary resuscitation is associated with delays in chest compressions. Resuscitation 119:95-8 